\title{
Calidad del contenido sobre la malaria en blogs de viajes en español
}

\author{
Laura Santos Larrégola 1,2, Manuel Linares Rufo ${ }^{2,3, *}$ y José Manuel Ramos Rincón 4, \\ 1 Centro de Salud Buenos Aires. Madrid. \\ 2 Fundación iO, www.fundacionio.org \\ 3 Hospital Universitario Príncipe de Asturias, Alcalá de Henares (Madrid) \\ 4 Universidad Miguel Hernández, Alicante. \\ * Autor correspondencia: manuellinares@fundacionio.com ; ORCID id: https://orcid.org/0000-0002-7190-0984
}

DOI: https://doi.org/10.37536/RIECS.2021.6.S1.246

Resumen: Cada año se notifican unos diez mil casos importados de malaria en Europa; 80 por cada 100.000 viajes a áreas endémicas. La no utilización de quimioproflaxis en los viajeros sigue siendo su principal motivo. Los blogs de viajes han proliferado a lo largo y ancho de toda la red siendo un lugar muy común de consulta, a veces el único utilizado, antes de preparar un viaje a un área endémica. El objetivo de este trabajo ha sido analizar la información ofrecida sobre malaria en los principales blogs de viajes en español. Para ello se seleccionaron y analizaron los cien blogs de viajes en español con mayor impacto. Los resultados encontrados mostraron una muy baja calidad en la información suministrada por este tipo de publicaciones y los contenidos ofrecidos por lo general eran insuficientes, incompletos o lo que es más grave inadecuados, pudiendo alentar en muchos casos al usuario a no tomar las medidas preventivas adecuadas. Como ejemplo el que los denominados "influencers de viajeros" comuniquen información fiable, contrastada y de calidad sobre malaria en sus canales puede contribuir a una reducción de la carga de la enfermedad en nuestro entorno.

Palabras Clave: Malaria, Paludismo, Viajero, Turista, Blog, Redes sociales, Consejo viajero, Quimioprofilaxis, Prevención.

\begin{abstract}
About 10,000 imported cases of malaria are reported in Europe each year; 80 for every 100,000 trips to endemic areas. The non-use of chemoprophylaxis in travelers continues to be its main reason. Travel blogs have proliferated throughout the entire network, being a very common place to consult, sometimes the only one used, before preparing a trip to an endemic area. The objective of this work was to analyze the information offered on malaria in the main travel blogs in Spanish. To do this, we selected and analyzed 100 travel blogs that were considered highly relevant by its metrics. The results we found show a very low quality in the information provided by this type of publications. The content they offer is often insufficient, incomplete or what is worse, inadequate. They can confuse the user into not following proper preventive measures. The so-called "travel influencers" must communicate reliable, verified and quality information on malaria on their channels in a way that could contribute to a reduction in the burden of the disease in our environment.
\end{abstract}

Key words: Malaria, Tourist, Travellers, Blog, Social networks, Travel advice, Chemoprophylaxis, Prevention. 


\section{Introducción}

La malaria representa un grave peligro para la salud de los viajeros. Cada año se notifican aproximadamente cien mil casos importados en Europa; 80 por cada 100.000 viajes a áreas endémicas. La incidencia global de la enfermedad se estimó en 229.000 .000 casos en 2019, de los cuales más del $50 \%$ se produjeron en África [1,2].

A pesar de disponer de dos bases de datos oficiales (Red Nacional de Vigilancia Epidemiológica [RENAVE] y Conjunto Mínimo Básico de Datos [CMBD], ingresos hospitalarios) la carga real de malaria importada en nuestro país parece estar subestimada [3,4]. Existe además una gran limitación para el cálculo de su prevalencia al solo disponer de encuestas y no del número real de viajeros por origen y destino [5].

Las cifras de casos ofrecidas por el Centro Europeo para la Prevención y Control de Enfermedades (ECDC) para España muestran una tendencia creciente los últimos años: 688 casos (año 2014), 706 (año 2015), 755 (año 2016), 818 (año 2017) y 851 (año 2018) [6] Datos muy probablemente relacionados con el aumento de viajeros a zonas de riesgo. En 2018 un 9,8\% de los 197,5 millones de viajes de los españoles fue al extranjero [5,7]. Se sabe que el riesgo de malaria importada es mayor en los viajeros de África, especialmente los inmigrantes y los visiting friend and relatives (VFRs). Son muchos los estudios que señalan que la no utilización de quimioproflaxis sigue siendo demasiado común entre los casos de malaria importados, particularmente entre estos grupos y otros menos esperados como jóvenes o viajeros con un nivel educativo alto $[8,9]$.

Buscando el origen de dicho comportamiento, al ya conocido consejo previaje que debe realizarse de forma individualizada, en función de los antecedentes culturales, la ruta de viaje exacta, la temporada o el tipo de viaje proponemos un análisis más detallado sobre la influencia de las fuentes "no oficiales" de información o consulta como los blogs de viajes en la actitud del viajero. Los blogs de viajes han proliferado a lo largo y ancho de toda la red y hoy en día existen miles, y cada año se crearán más. Los viajeros aprenden de ellos o buscan información concreta para próximos destinos existiendo portales digitales específicos por destinos, para mochileros, viajeros de negocios, viajeros más jóvenes o aquellos que viajan por períodos de tiempo más largos.

A la vista del número de visitas que tienen, muy superiores a las páginas webs oficiales de los organismos sanitarios que proporcionan consejo, hacen que este tipo de blogs se hayan convertido en los lugares favoritos de los viajeros para buscar consejos y recomendaciones cuando se prepara una ruta nueva [10].

Así, el objetivo de este trabajo es analizar la información ofrecida sobre malaria en los principales blogs de viajes en español, abriendo el camino para un asesoramiento basado en "la buena información en redes" para la prevención del riesgo de malaria entre los viajeros.

\section{Material y Métodos}

\subsection{Tipo de estudio y fuente de obtención de los datos}

Estudio descriptivo transversal en la que la población del estudio fueron cien blogs de viajes de contenido general en español entre más de 500 consultados (Búsqueda obtenida en Google). La selección se hizo en base a las métricas obtenidas con las siguientes herramientas externas: i) redes sociales (RRSS) midiendo el total de seguidores de Facebook, Twitter, Instagram y YouTube), utilizando como herramientas de medidas Influencer Marketing Hub, Fanpage Karma y Metricool, ii) número de visitas mensuales utilizando la herramienta SEMrush, iii) nutoridad del dominio (DA), utilizando la herramienta MOZ.y iv) número de backlinks o enlaces entrantes teniendo en cuenta la edad y el porcentaje según categoría. La herramienta utilizada en este caso también fue SEMrush. 


\subsection{Tratamiento de la información}

Se utilizó el buscador del propio blog para encontrar contenidos que incluyeran la palabra "malaria o paludismo", también se incluyeron los formatos de video y podcast. Para la evaluación de la calidad de la información vertida los diferentes posts encontrados se respondieron a las siguientes preguntas con respuestas dicotómicas: 1) ¿Ofrece el blog información sobre malaria? (Si/No). 2) ¿Ofrece el blog enlaces a webs oficiales/especializadas en medicina del viajero? ( $\mathrm{Si} / \mathrm{No}$ ) en este caso se anotaron cuáles. 3) ¿Dispone el blog de información adecuada en materia de quimioprofilaxis (Si/No). 4) La información que proporciona ¿está actualizada? Entendida tal como que el contenido ha sido revisado en los últimos 12 meses (Si/No). 5) La información que proporciona iestá referenciada? (Si/No) entendiendo un no como meras opiniones personales. 6) ¿Aconseja el blog productos preventivos frente a la malaria no certificados o cuya eficacia esté en cuestión? *ver listado (Si/No) 7) ¿Proporciona información correcta sobre el autotratamiento (SBET "stanby emergency treatment")? (Si/No) 8) ¿Recoge información sobre los efectos secundarios de la quimioprofilaxis? (Si/No) en este ítem se hizo un análisis cualitativo de la información proporcionada. 9) ¿Hay línea de discusión sobre la malaria en el foro o comentarios a los posts? (Si/No) 10) La información ofrecida sobre malaria ¿ha sido generada o revisada por un profesional sanitario? (Si/No)

- Listado de productos preventivos no certificados o cuya eficacia esté en cuestión: Ajo, cebolla, vitamina B, pulseras, óxido nitroso, diversos cosméticos, velas antimosquitos, dispositivos eléctricos o ultrasónicos como repelentes de mosquitos.

\section{Resultados}

De los 100 blogs analizados se encontró que un 36\% de ellos no proporcionaba ningún tipo de información respecto a la malaria aun siendo páginas que ofrecían recomendaciones para visitar países endémicos. Paradójicamente un 95\% sí ofrecían publicidad o contenido publicitario sobre seguros médicos para viajar.

De los 64 que sí ofrecían información solo 10 proporcionan enlaces a webs oficiales/especializadas (Centros de vacunación internacional 5, Ministerio de Sanidad 2, otras páginas webs especializadas dedicadas a la medicina de viajes 3 ).

Tabla I Ítems de calidad de los blogs analizados

\begin{tabular}{|l|c|c|}
\hline \multicolumn{1}{|c|}{ Ítems de calidad } & $\mathrm{N}$ & $\%$ \\
\hline 1) ¿Ofrece el blog información sobre malaria? & 100 & $64 \%(64)$ \\
\hline $\begin{array}{l}\text { 2) ¿Ofrece el blog enlaces a webs oficiales/especializadas en medicina } \\
\text { del viajero? }\end{array}$ & 64 & $15,62 \%(10)$ \\
\hline $\begin{array}{l}\text { 3) ¿Dispone el blog de información adecuada en materia de } \\
\text { quimioprofilaxis? }\end{array}$ & 64 & $10,93 \%(7)$ \\
\hline 4) La información que proporciona ¿está actualizada? & 64 & $34,37 \%(22)$ \\
\hline 5) La información que proporciona iestá referenciada? & 64 & $6,25 \%(4)$ \\
\hline $\begin{array}{l}\text { 6) ¿Aconseja el blog productos preventivos frente a la malaria no } \\
\text { certificados o cuya eficacia esté en cuestión? }\end{array}$ & 64 & $15,62 \%(10)$ \\
\hline $\begin{array}{l}\text { 7) ¿Proporciona información correcta sobre el autotratamiento SBET } \\
\text { "stanby emergency treatment"? }\end{array}$ & 64 & $3,12 \%(2)$ \\
\hline $\begin{array}{l}\text { 8) ¿Recoge información sobre los efectos secundarios de la } \\
\text { quimioprofilaxis? }\end{array}$ & 64 & $68,75 \%(44)$ \\
\hline $\begin{array}{l}\text { 9) ¿Hay línea de discusión sobre la malaria en el foro o comentarios a } \\
\text { los post? }\end{array}$ & 64 & $75 \%(48)$ \\
\hline $\begin{array}{l}\text { 10) La información ofrecida sobre malaria ¿ha sido generada o revisada } \\
\text { por un profesional sanitario? }\end{array}$ & 64 & $3,12 \%(2)$ \\
\hline
\end{tabular}


En el análisis cualitativo de los blogs fueron detectados los siguientes errores de concepto importantes y con trascendencia en el consejo:

- En 12 de ellos se recomendaba "la vacuna de la malaria" para viajar. Clara confusión con el concepto de quimioprofilaxis.

- La posología de los antimaláricos en muchos casos era errónea o su indicación estaba desactualizada (se hablaba por ejemplo del uso de la cloroquina en zonas de actual resistencia).

- En algunos de los blogs se recomendaban productos basados en vitamina B o repelentes y dispositivos frente a mosquitos de dudosa eficacia y científicamente no probados.

La descripción de efectos secundarios de la quimioprofilaxis aparece en 44 blogs. Destacamos en algunos de ellos palabras como: "veneno, graves complicaciones, pastillas agresivas o brote psicótico".

\section{Discusión}

Son conocidos los esfuerzos ya iniciados en prevención frente a la malaria en otros colectivos como agentes de viajes o en contextos muy concretos de viajeros [11-13] Sin embargo en el campo de los blogs de viajes estas iniciativas hasta el momento son prácticamente inexistentes. Más en el contexto actual de infoxicación o presencia de fake news que presentan las redes [14].

Se podría establecer unos "criterios filtro" en los blogs de viajes y dejar al usuario que sea el que evalúe la información obtenida. Al igual que ocurre en otras fuentes de información, denominadas actualmente "clásicas", como periódicos, radio o televisión, donde los mensajes y su contenido pueden ser erróneos, incompletos o presentar un punto de vista totalmente sesgado, y no por ello se dejan de utilizar, siendo el usuario quien forma su juicio personal. Posiblemente, la existencia de códigos de conducta y ética, como HONcode [15], puedan ser una manera más fiable de la existencia de una información de calidad y creíble, ya que una proporción significativa de personas tiene poca alfabetización en salud, y las personas con poca alfabetización en salud tienen dificultades para acceder a esta información, evaluar su calidad y aplicarla a sus propias circunstancias [16].

\section{Conclusiones}

En consecuencia, se podría concluir que existe una baja calidad del blog de viajes en español respecto a la malaria. La calidad científica de los blogs puede tener una relación con un mayor número de casos de malaria importada y de casos graves de la misma, ya que el viajero toma el consejo de la información de los mismos sin estar bien documentada ni avalada por la opinión de expertos en la materia.

Mejorar la adherencia en viajeros.

Las percepciones de los viajeros sobre los riesgos de la malaria deben sean realistas y corregir las percepciones erróneas (como creer que curar la malaria es más fácil que tomar profilaxis o que los viajeros que visitan a familiares tienen algún nivel de inmunidad innata).

Agradecimientos: Las contribuciones e ideas de todos los blogueros de viajes consultados.

Contribución de los autores: Todos los autores han contribuido por igual al manuscrito. Todos los autores han leído y aprobado el manuscrito.

Conflictos de Intereses: Los autores declaran no haber recibido financiación para la realización de este estudio 


\section{Abreviaturas}

Las siguientes abreviaturas son usadas en este manuscrito:

SBET: Stanby emergency treatment

CMBD: Centralized Hospital Discharge Database

ECDC: Europan Centers for Disease Control and Prevention

DA: Autoridad de dominio (Domain Authority)

INE: Instituto Nacional de Estadística

RENAVE: National Network of Epidemiological Surveillance

RRSS: Redes sociales

SEO: Search Engine Optimization (Optimizacion en Motores de Búsqueda)

SSL: Secure Sockets Layer o capa de conexión segura

VFRs: visiting friends and relatives

\section{Referencias bibliográficas}

1. Arguedas-Arguedas O. Tipos de diseño en estudios de investigación biomédica. Acta Med Costarric [Internet]. 2010 [Consultado el 15 de abril 2016]; 52 (1): 16-18. Disponible en: http://www.scielo.sa.cr/scielo.php?script=sci_arttext\&pid=S0001-60022010000100004\&lng=en.

2. (1b) WHO. World malaria report 2020. Geneva: World Health Organization; 2020. https://www.who.int/publications/i/item/9789240015791. Acceso 1 Feb 2021.

3. Herrador, Z., Fernández-Martinez, B., Quesada-Cubo, V. et al. Imported cases of malaria in Spain: observational study using nationally reported statistics and surveillance data, 2002-2015. Malar J 18, 230 (2019). https://doi.org/10.1186/s12936-019-2863-2

4. Tatem AJ, Jia P, Ordanovich D, Falkner M, Huang Z, Howes R, et al. The geography of imported malaria to non-endemic countries: a meta-analysis of nationally reported statistics. Lancet Infect Dis. 2017;17:98-107.

5. Instituto nacional de Estadística (INE). España en cifras. 2019. https://www.ine.es/prodyser/espa_cifras/2019/51/ Acceso 1 Feb 2021.

6. European Centre for Disease Prevention and Control. Introduction to the Annual Epidemiological Report [internet]. Stockholm: ECDC; $2020 . \quad$ Disponible en: https://www.ecdc.europa.eu/en/publications-data/malaria-annual-epidemiological-report-2018

7. Instituto nacional de Estadística. INE. https://www.ine.es/ Acceso 1 Feb 2021.

8. Hoefnagel JGM, Massar K, Hautvast JLA. Non-adherence to malaria prophylaxis: The influence of travel-related and psychosocial factors. J Infect Public Health. 2020 Apr;13(4):532-537. doi: 10.1016/j.jiph.2019.10.004. Epub 2019 Nov 6. PMID: 31704047.

9. Ahluwalia J, Brooks SK, Weinman J, Rubin GJ. A systematic review of factors affecting adherence to malaria chemoprophylaxis amongst travellers from non-endemic countries. Malar J. 2020 Jan 13;19(1):16. doi: 10.1186/s12936-020-3104-4. PMID: 31931813; PMCID: PMC6958680.

10. Alexa. https://www.alexa.com/topsites/countries/ES

11. Heimdllr Project. ¿De qué mueren los turistas? https://fundacionio.com/desarrollo-io/heimdllrproject/de-que-mueren-los-turistas/

12. Bazaz R, Green E, Green ST. Quality of malaria information provided on Internet travel operator websites. Travel Med Infect Dis. 2010 Sep;8(5):285-91. doi: 10.1016/j.tmaid.2010.07.005. PMID: 20971438.

13. Ozan-Rafferty ME, Johnson JA, Shah GH, Kursun A. In the words of the medical tourist: an analysis of Internet narratives by health travelers to Turkey. J Med Internet Res. 2014 Feb 6;16(2):e43. doi: 10.2196/jmir.2694. PMID: 24513565; PMCID: PMC3936263. https://www.jmir.org/2014/2/e43/

14. Giandomenico Di Domenico, Jason Sit, Alessio Ishizaka, Daniel Nunan, Fake news, social media and marketing: A systematic review, Journal of Business Research, Volume 124, 2021, Pages 329-341, ISSN 0148-2963, https://doi.org/10.1016/j.jbusres.2020.11.037 . 
15. Health On the Net Foundation (HON Foundation). The HON Code of Conduct for medical and health Web sites (HONcode) [monograph on the Internet]. Geneva, Switzerland: HON Foundation; 2017 [cited October 14, 2019]. Available in: http://bit.ly/2PpHUcj

16. Charow R, Snow M, Fathima S, Giuliani ME, McEwan K, Winegust J, et al. Evaluation of the scope, quality, and health literacy demand of Internet-based anal cancer information. J Med Libr Assoc JMLA. 2019;107(4):527-37. DOI: 10.5195/jmla.2019.393

(C) 2021 por los autores; Esta obra está sujeta a la licencia de Reconocimiento 4.0

Internacional de Creative Commons. Para ver una copia de esta licencia, visite http://creativecommons.org/licenses/by-nc-nd/4.0/. 